\title{
Nota corta \\ Dinámica de las terrazas fluviales en la subcuenca Grijalva-Villahermosa, México
}

\author{
Neiser Zamora Saud, Eunice Pérez Sánchez, Víctor Roberto Carballo Gruz, \\ Adalberto Galindo Alcántara
}

\begin{abstract}
Neiser Zamora Saud Eunice Pérez Sánchez

Víctor Roberto Garballo Gruz Adalberto Galindo Alcántara eperezsanchez@netscape.net

Universidad Juárez Autónoma de Tabasco, División Académica de Ciencias Biológicas. Carretera Villahermosa-Cárdenas Km. 0.5 entronque a Bosques de Saloya. 86150 Villahermosa, Tabasco, México.
\end{abstract}

BOL. SOC. GEOL. MEX. 2018

VOL. 71 NO. 3

P. $805-817$

http://dx.doi.org/10.18268/BSGM2019v71n3a10

Manuscrito recibido: Octubre 09, 2017.

Manuscrito corregido: Febrero 05, 2018.

Manuscrito aceptado: Febrero 9, 2018.

\section{RESUMEN}

El análisis geoespacial, mediante estudios comparativos de imágenes satelitales a través de un sistema de información geográfica, ha permitido poner de manifiesto los cambios territoriales generados por la interacción entre procesos morfológicos naturales y actividades antropogénicas. La subcuenca GrijalvaVillahermosa forma parte de una de las cuencas más grandes de México. Por la ubicación geográfica y características geológicas del Río Grijalva está constituida por sedimentos del cuaternario reciente holoceno aluvial. La geomorfología corresponde a planicie fluviodeltaica con pendiente casi plana, por lo que las inundaciones son constantes. Con la finalidad de evaluar el efecto de los sistemas de control de agua en la dinámica de las terrazas fluviales se desarrolló un diagnóstico ambiental, tomando en cuenta el efecto de obras de mitigación de inundaciones. Las terrazas fluviales fueron identificadas mediante análisis espectral de imágenes LiDAR de 2011 y verificadas a través de fotointerpretación y visitas en campo. El diagnóstico ambiental se basó en imágenes Landsat de 1986 y 2016, a las cuales se aplicó el análisis de tendencia de cambio con cadenas de Markov. El criterio ambiental del análisis fue basado en la evaluación del cambio en la cobertura de humedales, vegetación, infraestructura antrópica, cuerpos de agua y suelo. Los resultados en la dinámica de las terrazas fluviales más significativos en términos de ganancia de superficie se registraron en humedales con 13034 ha y una pérdida de 6475 ha, en infraestructura antrópica ganando 3242 ha y perdiendo 3793 ha, así como una ganancia de 1145 ha y una pérdida de 3519 ha en cuerpos de agua. Los resultados mostraron una tendencia de cambio en la cobertura de humedales de forma intensiva en el periodo de análisis. La dinámica actual en la Subcuenca muestra un comportamiento regular en la formación de las terrazas fluviales, pero un impacto negativo debido a factores antropogénicos.

Palabras clave: Impacto antropogénico, inundaciones, tendencias de cambio, uso de suelo

\section{ABSTRACT}

Geospatial analysis, based on comparative studies of satellite images through geographical information systems, has allowed to highlight territorial changes generated by the interaction between natural morphological processes and anthropogenic activities. The Grijalva-Villahermosa sub-basin is part of one of the largest watersheds in Mexico, the Grijalva-Usumacinta basin. Based on geographical location and geological characteristics Grijalva River is formed by sediments of recent quaternary alluvial Holocene. Geomorphology corresponds to the fluviodeltaic plain with an almost flat slope, so floods are constant. In order to evaluate the effect of water control systems in the dynamics of the fluvial terraces an environmental assessment was developed, taking into account the effect of flood mitigation structures. The fluvial terraces were identified through spectral analysis of 2011 LiDAR images and verified through photo-interpretation and field visits. The environmental assessment was based on Landsat images 1986 and 2016, to which the analysis of change trend with Markov chains was applied. The environmental criterion of the analysis was based on the evaluation of change on the vegetation coverage, wetlands, water bodies, soil and anthropic infrastructure. The most significant results on the dynamics of the fluvial terraces in terms of surface gain occurred in wetlands with 13034 ha and a loss of $6475 \mathrm{ha}$, in anthropic infrastructure gaining was 3242 ha and losing 3793 ha, as well as a gain of 1145 ha and a loss of 3519 ha in water bodies. The results showed an intensive change trend in wetlands coverage on the analysis period. The current dynamics in the Subbasin shows a regular behavior in the formation of the fluvial terraces, but a negative impact due to anthropic factors.

Keyzords: Anthropic impact, floods, change trends, land use. 


\section{Introducción}

La influencia de las vías de agua, definidas preferentemente por la acción directa del río, integra a importantes unidades cuya morfogénesis y dinámica tienen diversos factores de control. Así mismo, el contexto geomorfológico del dominio fluvial, en sentido amplio, puede asumirse también como una de las claves para entender, por ejemplo, la evolución de la llanura de inundación, las modificaciones del trazado del cauce, o incluso, la importancia y frecuencia de los desbordamientos (Schumm, 1993; Howard, 1996).

Los sistemas de terrazas fluviales son considerados como la antigua llanura de inundación de un río que es "abandonada" a un nivel superior del actual nivel de la llanura de inundación. Ésta es un sistema complejo en el que se incluyen formas asociadas al canal, características del borde (depósitos laterales, diques) y características de la propia llanura de inundación, como antiguos canales y depósitos (Brown, 1996). Por otro lado, en ella convergen factores de relación compleja, como la variabilidad de los regímenes energéticos que son reflejo de los procesos hidrológicos y sedimentológicos (Nanson y Croke, 1992), lo cuales manifiestan circunstancias ambientales tradicionalmente identificadas como factores de control de formación de terrazas fluviales (geología, clima, vegetación, usos del suelo, entre otros). En este sentido, las planicies de inundación son ricas en nutrimentos debido a que cuando una corriente de agua escapa de sus bancos, deja en ellas un depósito que, con el tiempo, forma un rico suelo aluvial.

Una de las formas de evaluar la dinámica de uso del suelo es la fotointerpretación de imágenes aéreas y el análisis de imágenes de satélite (Vidal y Romero, 2010). De esta manera los cambios de uso del suelo pueden evaluarse a través de la comparación histórica, detectada por el uso de fotografías aéreas de diferentes años (Ortiz-Solorio et al., 1995; Ramos-Reyes, 2004; Rojas et al., 2014), los cuales se interpretan y se vacía la información en mapas cartográficos que pueden manipularse utilizando los sistemas de información geográfica (SIG).
Los modelos de cambio de uso de suelo recientemente se han considerado como herramientas importantes para analizar las causas y consecuencias de la dinámica de los sistemas, como es el caso de los que corresponden a los sistemas de control de agua, que son derivados de factores antrópicos, y tienen un impacto sobre los ecosistemas terrestres, la pérdida y la fragmentación de hábitat, así como impactos negativos sobre la vida humana.

El área de estudio, de acuerdo con Zavala-Cruz et al. (2016) predominan dos paisajes geomorfológicos: Planicie Fluvial Activa y Terrazas Costeras, los cuales se constituyen por la acumulación de sedimentos del cuaternario holoceno dando lugar a la región fisiográfica Llanuras Costeras del Golfo Sur donde se encuentra la Cuenca GrijalvaUsumacinta. Ésta presenta una gran diversidad de regiones ecogeográficas: planicies inundables, terrazas fluviales y terrazas aluviales, valles, colinas y laderas. Por ello, la gestión o manejo adecuado de planicies y áreas inundables debe dirigirse a reducir los efectos destructivos de las crecidas, a preservar y enaltecer los valores naturales y proveer criterios para la utilización óptima de los recursos terrestres y acuáticos de las planicies inundables. Existen diversos estudios que tratan el tema de terrazas fluviales bajo distintos enfoques: geomorfológico, geopedológico, hidrológico, biológico, geológico, entre otros (Ortiz-Perez et al., 2005; Vidal y Romero, 2010; Ortiz-Solorio et al., 1995; Zavala-Cruz et al., 2014; Zinck, 2016). Los elementos de análisis integral considerado para esta investigación surgen de fundamentos geomorfopedológicos con un enfoque ambiental, en el que se considera al ambiente como un sistema complejo con subsistemas interrelacionados. De acuerdo con Zinck (2016), las geoformas pueden ser percibidas por medio de sensores remotos, ya que representan una apariencia fisionómica de la superficie terrestre. De esta manera, las geoformas son los elementos estructurales más directos del terreno, como en el caso del relieve, que permite al observador determinar sus componentes. Por lo tanto, el objetivo fue evaluar el efecto de los sistemas de control en la dinámica de las terrazas fluviales en la Subcuenca Grijalva-Villahermosa. 
Dicha evaluación se basó en un modelo SIG de tendencias de cambio para identificar patrones antecedentes y actuales en los procedimientos de mitigación de riesgos de inundación y en la planeación del aprovechamiento del suelo en zonas identificadas como de bajo riesgo en los programas de ordenamiento territorial estatal y municipal.

\section{Descripción del área de estudio}

La cuenca Río Grijalva-Villahermosa es una de las más grandes de México y más importante de Tabasco (Toledo Sánchez, 2011). Su corriente principal se origina en territorio guatemalteco, teniendo su origen por la unión de los ríos San Gregorio y San Miguel, los cuales cruzan por separado la frontera entre México y Guatemala. Al unirse en nuestro país constituyen el Río Grande de Chiapas o Mezcalapa en el Alto Grijalva. El área de estudio se concentra en los municipios del Centro y Gunduacán, colindando al norte con el municipio de Comalcalco, al sur con el Estado de Chiapas, al oeste con Cárdenas y al este con Jalpa, Nacajuca y Centro.

De acuerdo a la síntesis de información geográfica del estado de Tabasco (INEGI, 2001) la zona del proyecto se encuentra en la Región Hidrológica 30: Cuenca Río Grijalva - Villahermosa (D), constituida por la Subcuenca Río Viejo Mezcalapa (Db) y Subcuenca Río Samaria (Dx) (Figura 1).

Los principales recursos hidrológicos del municipio son las aguas del río Grijalva con sus afluentes: el Río Samaria, que tiene sus orígenes en el municipio de Huimanguillo y es una Subcuenca con sistemas de control de inundaciones. El Río Mezcalapa, que se bifurca antes de llegar a la ciudad de Cunduacán y es un Subcuenca en la que no se ha desarrollado ningún sistema de control.

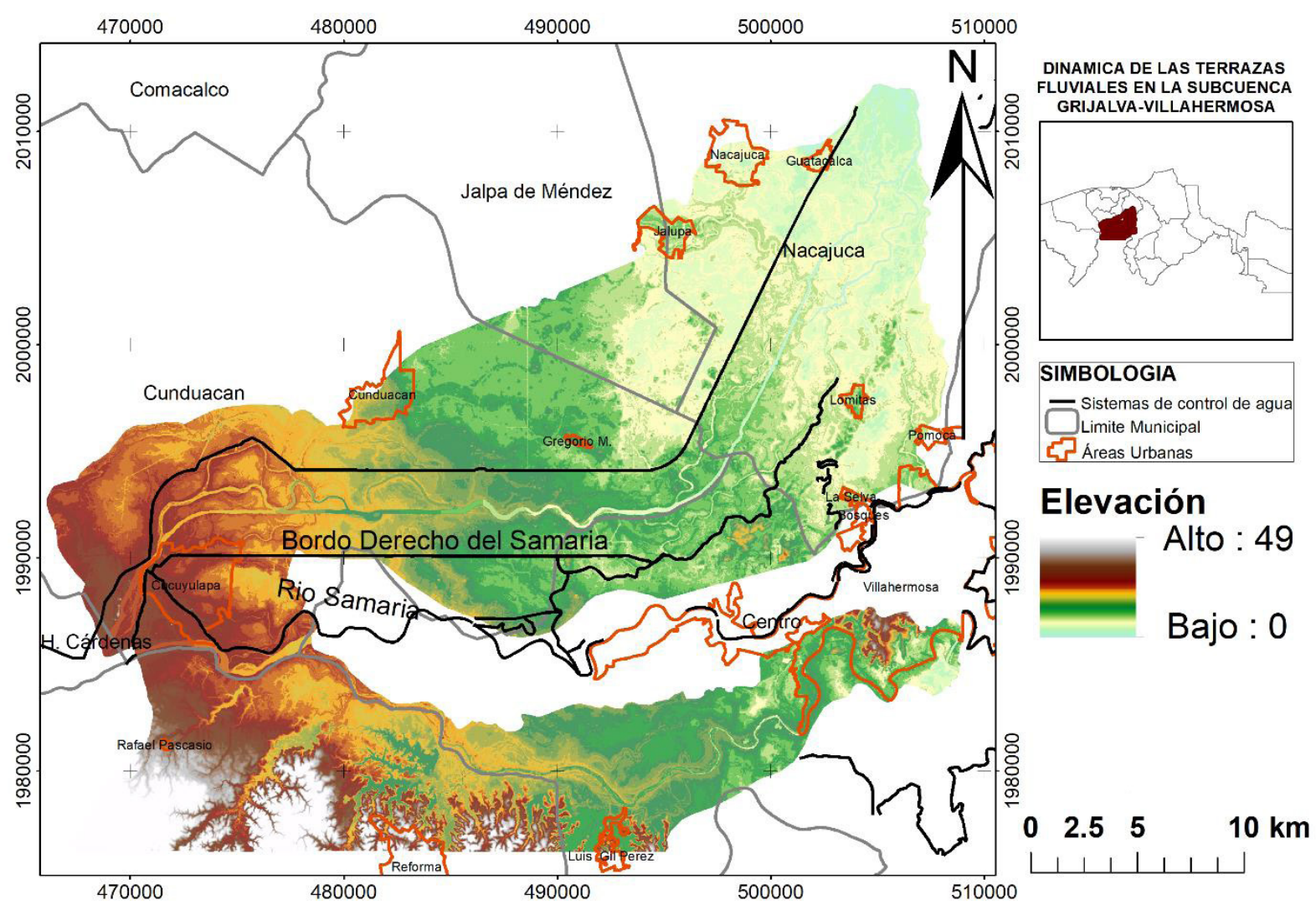

Figura 1 Localización del área de estudio. 


\section{Materiales y métodos}

La caracterización de las terrazas fluviales se llevó a cabo mediante la integración de información cartográfica (suelos, geología, vegetación, uso del suelo, geomorfología). El modelo digital de elevación (MDE) se desarrolló a partir de datos LiDAR tipo terreno a $5 \mathrm{~m}$ de resolución, que fueron proporcionados por el INEGI.

El relieve se clasificó de acuerdo al enfoque geopedológico en el ambiente morfogenético originado y controlado por un estilo de geodinámica interna y/o externa, estructural y deposicional con base en el MDE (reclasificación) y ortofotos digitales 2008 (fotointerpretación) de la Subcuenca del Río Samaria (cartas E15A88, E15A89 y E15B81) y para la Subcuenca del Río Mezcalapa (cartas E15C19 y E15D11), a escala 1: 220000 con el programa ArcGIS. La diferenciación de los paisajes geomorfológicos se realizó con información morfométrica de forma, altitud, pendiente, proceso geomorfológico y tipo de roca, de acuerdo con Ortíz-Pérez et al. (2005) y Zavala-Cruz et al. (2016).

Los criterios ambientales (humedales, cuerpos de agua, vegetación, suelo e infraestructura antrópica) para el desarrollo del diagnóstico ambiental de la dinámica de las terrazas fluviales fueron identificados por medio de una clasificación no supervisada de las imágenes Landsat de 1986 Enhanced Thematic Mapper (ETM) y 2016 Operational Land Imager (OLI) con una resolución de 15 m, las cuales fueron posteriormente reclasificadas para el análisis de tendencia de cambio (CA_MARKOV). El diagnóstico ambiental se basó en el medio natural y antrópico en la zona de estudio, que consistió en la evaluación del cambio en la cobertura de humedales (ecosistema), cuerpos de agua, vegetación (pastizales y cultivos), suelo e infraestructura antrópica (sistemas de control de agua y zonas urbanas), los cuales representan los sistemas ambientales característicos en la desembocadura de la Subcuenca Grijalva-Villahermosa. El relieve obtenido del proceso de reclasificación y fotointerpretación fue integrado en este paso del modelo SIG. La resolución espacial de las imágenes Landsat permitió diferenciar la heterogeneidad de comunidades vegetales en la clasificación de humedales (Barba-Macías et al. 2006; GuerraMartínez y Ochoa-Gaona, 2008).

El método de cadenas de Markov simula la predicción del estado de un sistema en un tiempo determinado a partir de dos estados precedentes. Mediante el programa Idrisi se aplicó el método como una función (CA_MARKOV) entre las categorías de uso de suelo identificadas para el diagnóstico ambiental. Esta función integra la modelización temporal, la cual se basa en la lógica de la relación entre las categorías de uso de suelo y un conjunto de variables de diversa naturaleza que pueden explicar o describir su dinámica, que en este trabajo están conformadas por los criterios ambientales. Con el propósito de facilitar la interpretación de los patrones de cambio se aplicó un polinomio cuadrático de ajuste de tendencia, el cual por de fault es de tercer grado en el módulo TREND $\left(\mathrm{ax}^{3}+\mathrm{bx}^{2}+\mathrm{cx}+\mathrm{d}=0\right)$ y es usada para determinar si existe la tendencia dentro del grupo de datos, es decir, en la cobertura analizada. De esta manera, el análisis multi-temporal (imágenes 1986 y 2016) se complementa con el análisis multi-variable (criterios ambientales) para obtener una modelización más ajustada a la dinámica real de los sistemas que permite estimar una matriz de probabilidad de transición (Paegelow et al., 2003; Requena, 2015). Las matrices de probabilidad y los mapas de probabilidad de transición para el análisis de tendencias de cambio se generaron con el módulo MARKOV de IDRISI usando las coberturas reclasificadas conforme a los criterios ambientales de 1986 (tiempo 1) y 2016 (tiempo 2). La proyección del uso del suelo mediante Markov parte del supuesto que la dinámica de los elementos espaciales resulta del estado actual del elemento o criterio, más un factor de proximidad aportado por el estado de sus vecinos inmediatos. La dinámica interna del sistema fue determinada por la probabilidad que tienen de transformación hacia otro tipo de cobertura, lo que se denomina tendencia de cambio. De la misma forma, se integró al análisis la cobertura correspondiente a Sistemas de Control de Inundaciones, lo cual permitió evaluar la dinámica de las terrazas fluviales en la Subcuenca Grijalva-Villahermosa (Figura 2). 


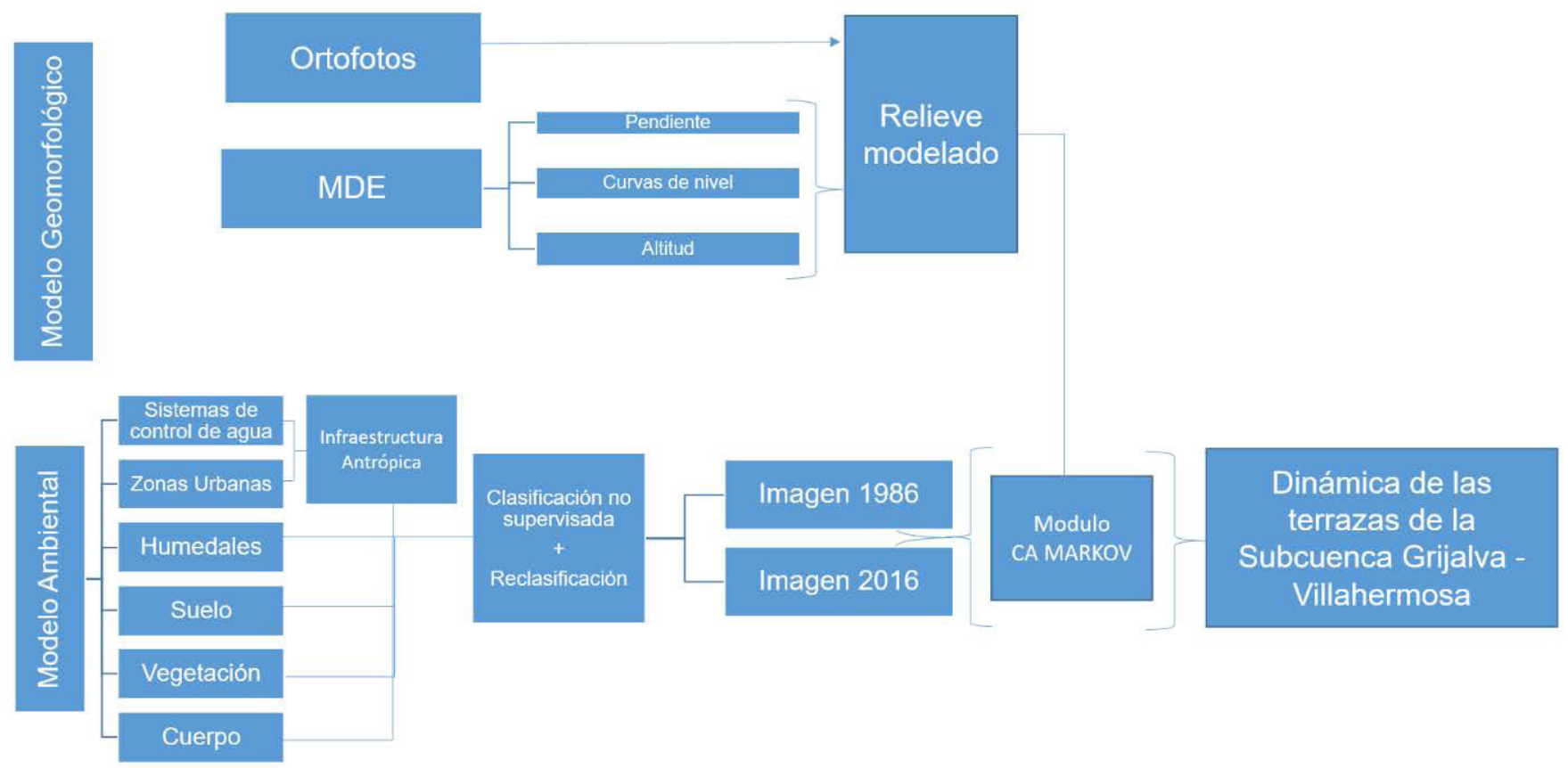

Modelación SIG de tendencias de cambio.

\section{Resultados}

\subsection{GARAGTERIZACIÓN DE LAS TERRAZAS FLUVIALES}

La caracterización de la dinámica de las terrazas fluviales permitió la identificación de cuatro diferentes niveles de terrazas (Figura 3), que varían en altura entre los 10 a 60 msnm y se observan asentamientos humanos de tipo rural y urbano:

1) Terraza baja plana-cóncava son terrazas bajas, el cual tiene una pendiente inferior a $1 \%$ y una altura entre 1 y 2 msnm. Las terrazas bajas se distribuyen al norte y noroeste de la Subcuenca Grijalva-Villahermosa, permanecen inundadas varios meses al año.

2) Terraza ligeramente ondulada que van de 8 a 13 msnm y sobresalen de la planicie fluvial como terrazas ligeramente onduladas con pendientes de 1 a $3 \%$, se inundan anualmente.

3) Terraza moderadamente ondulada se ubica en el suroeste del área de estudio, con alturas entre 14 y 25 msnm, pendientes de 5 a $10 \%$, coinciden con los asentamientos en la ciudad de Villahermosa y Luis Gil Pérez.
4) Terraza alta son mayor a 25 msnm con pendiente mayor a $10 \%$ las cuales se encuentran en la sierra de Chiapas.

Entre las terrazas se encuentran valles, los cuales se identificaron como: a) valles acumulativos de forma plana y alargada, reciben sedimentos proluviales aportados por arroyos que drenan las laderas, los cuales se inundan en la época de lluvias y b) valles erosivo-acumulativos que se forman por el proceso de erosión fluvial de las terrazas, tienen mayor pendiente que los acumulativos, son de forma cóncava y en las posiciones cercanas a la planicie fluvial, pueden inundarse en la época de lluvias.

\subsection{DIAGNÓSTICO AMBIENTAL DE LA DINÁMICA DE LAS TERRAZAS FLUVIALES}

Una vez que se ha establecido la caracterización de patrones que rigen la dinámica de las terrazas fluviales, se pueden explorar aquellos factores que influyen en estos. Desde el punto de vista de la dinámica de los sistemas ambientales, es posible desarrollar un sistema de información geográfica 


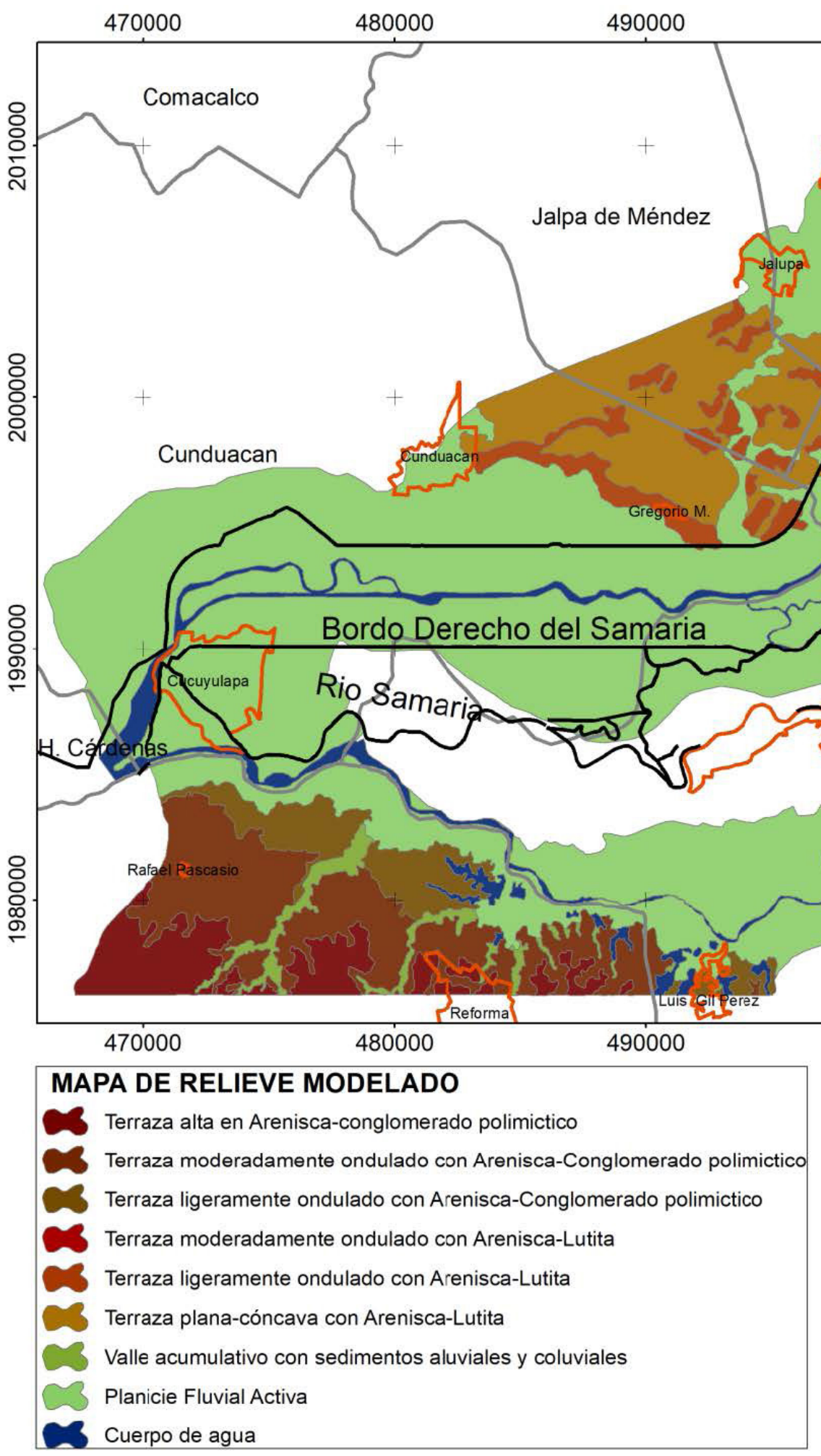


(Figura 2) que incorpore las terrazas fluviales (modelo geomorfopedológico) y los sistemas ambientales (modelo ambiental) para explicar el impacto de los sistemas de control de agua en la dinámica de la Subcuenca Grijalva-Villahermosa. En términos generales, se puede observar en la matriz de probabilidad de cambio (Tabla 1) que en la zona de estudio se registra una tendencia de cambio significativa en los cuerpos de agua (0.028) debido a su interacción con los sistemas de control de agua y zonas urbanas (infraestructura antrópica), es decir, el impacto de la infraestructura se ha visto incrementado en los procesos de retención de agua en los suelos impermeables. Estos cambios se han observado en zonas donde los periodos de inundación eran constantes y una vez establecidos los sistemas de control de agua estuvieron disponibles para diversas actividades antropogénicas. Por otra parte, la infraestructura antrópica ha funcionado como barrera, por lo que el relleno de áreas para la ampliación de las zonas urbanas se ha convertido en una práctica común, que favorece la ocupación del territorio en zonas inundables que representan por consecuencia zonas de riesgo de inundación. Los cambios generados por esta actividad incrementan el impacto que los sistemas de control de agua ejercen en otros sistemas ambientales. Se puede observar que la tendencia de cambio en humedales (0.023), suelos (0.225) y vegetación (0.205) es significativa por el cambio de uso de suelo.
El efecto que cada uno de los criterios ambientales ejerce sobre los demás criterios considerados en este trabajo se puede identificar mediante el análisis de los procesos de pérdidas y ganancias en superficie o terreno. En el caso de los cuerpos de agua, se puede observar en la Figura 4 que los cambios están asociados al impacto del desarrollo de los sistemas de control de agua y el aporte sobre los sedimentos debido al cambio de uso del suelo con una pérdida de 3519 ha. El total de hectáreas entre los cuerpos de agua que persistieron y las zonas que fueron ocupadas (2949 ha) por estos no rebasa el total de las pérdidas

En el caso de los humedales, el análisis de tendencia de cambio registró una pérdida de 6475 ha en el periodo de análisis (Figura 5). Sin embargo, en términos de ganancias se registraron 13035 ha de ocupación. Aunque esto puede apreciarse como un efecto positivo, las pérdidas registradas son el resultado negativo de los sistemas de control de agua, ya que los humedales prosperan en la medida que las geoformas correspondientes a la Terraza baja plana-cóncava se forman con suelos anegables.

Si se comparan los cambios entre la cobertura de humedales (Figura 5) y vegetación (Figura 6), en la zona norte de la Subcuenca se observa una tendencia de cambio hacia infraestructura antrópica de forma intensiva durante el periodo analizado debido al cambio de uso de suelo. El comportamiento opuesto, es decir, en términos de ganancia

Tabla 1. Probabilidad de Cambio CA MARKOV al 2017.

\begin{tabular}{|c|c|c|c|c|c|}
\hline \multirow[b]{2}{*}{ Criterios ambientales } & \multicolumn{5}{|c|}{ Probabilidad de cambio a: } \\
\hline & $\begin{array}{c}\text { INFRAESTRUCTURA } \\
\text { ANTRÓPICA }\end{array}$ & HUMEDALES & SUELO & CUERPO DE AGUA & VEGETACIÓN \\
\hline $\begin{array}{c}\text { INFRAESTRUCTURA } \\
\text { ANTRÓPICA }\end{array}$ & 0.5163 & 0.0238 & 0.2255 & 0.0287 & 0.2057 \\
\hline HUMEDALES & 0.0033 & 0.3050 & 0.2539 & 0.0035 & 0.4344 \\
\hline SUELO & 0.0239 & 0.1644 & 0.3315 & 0.0004 & 0.4797 \\
\hline CUERPO DE AGUA & 0.0025 & 0.0176 & 0.0436 & 0.6844 & 0.2519 \\
\hline VEGETACIÓN & 0.037 & 0.1764 & 0.2792 & 0.0092 & 0.4982 \\
\hline
\end{tabular}




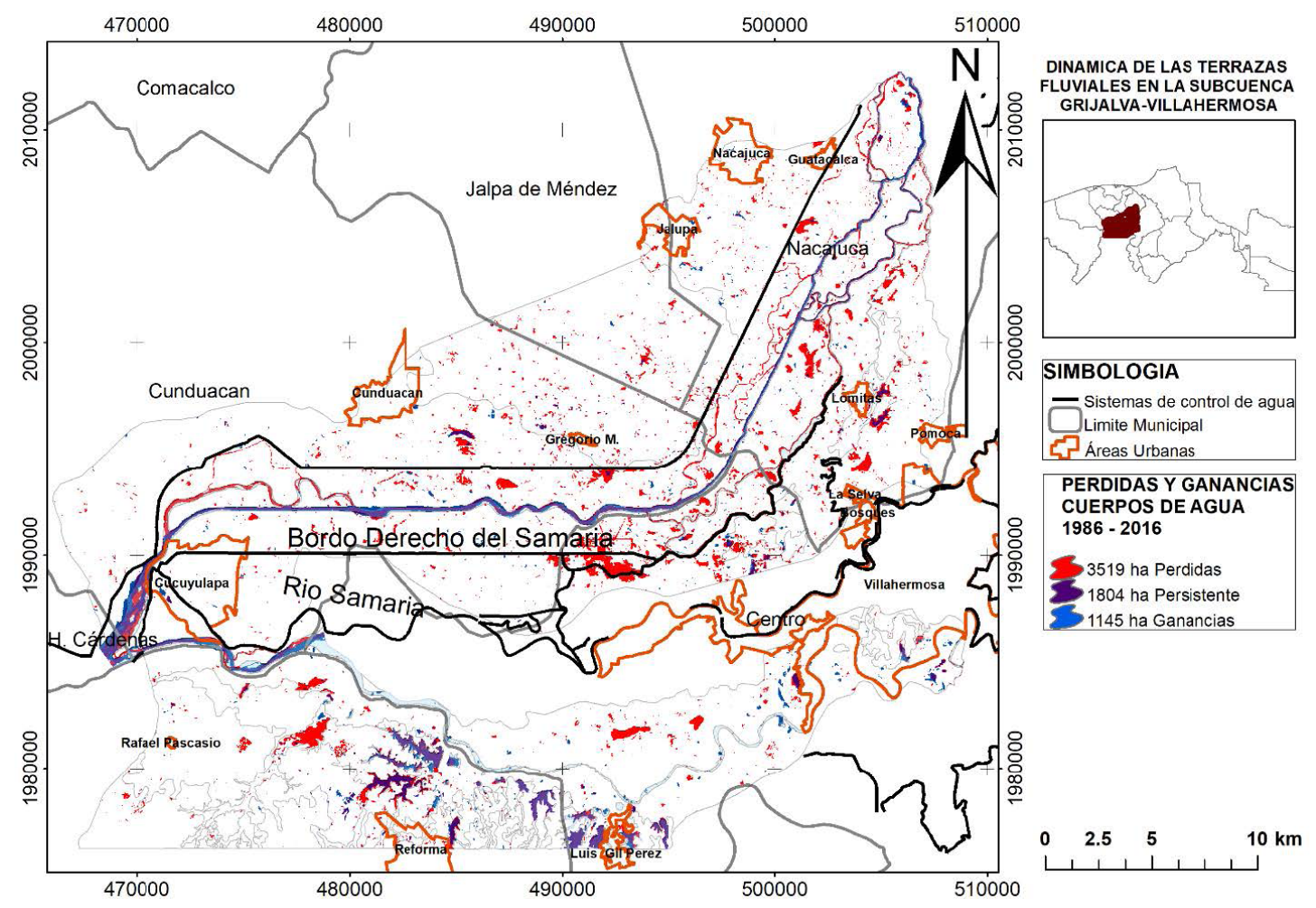

Figura 4 Mapa de pérdidas y ganancias de Cuerpos de Agua del periodo 1986-2016.

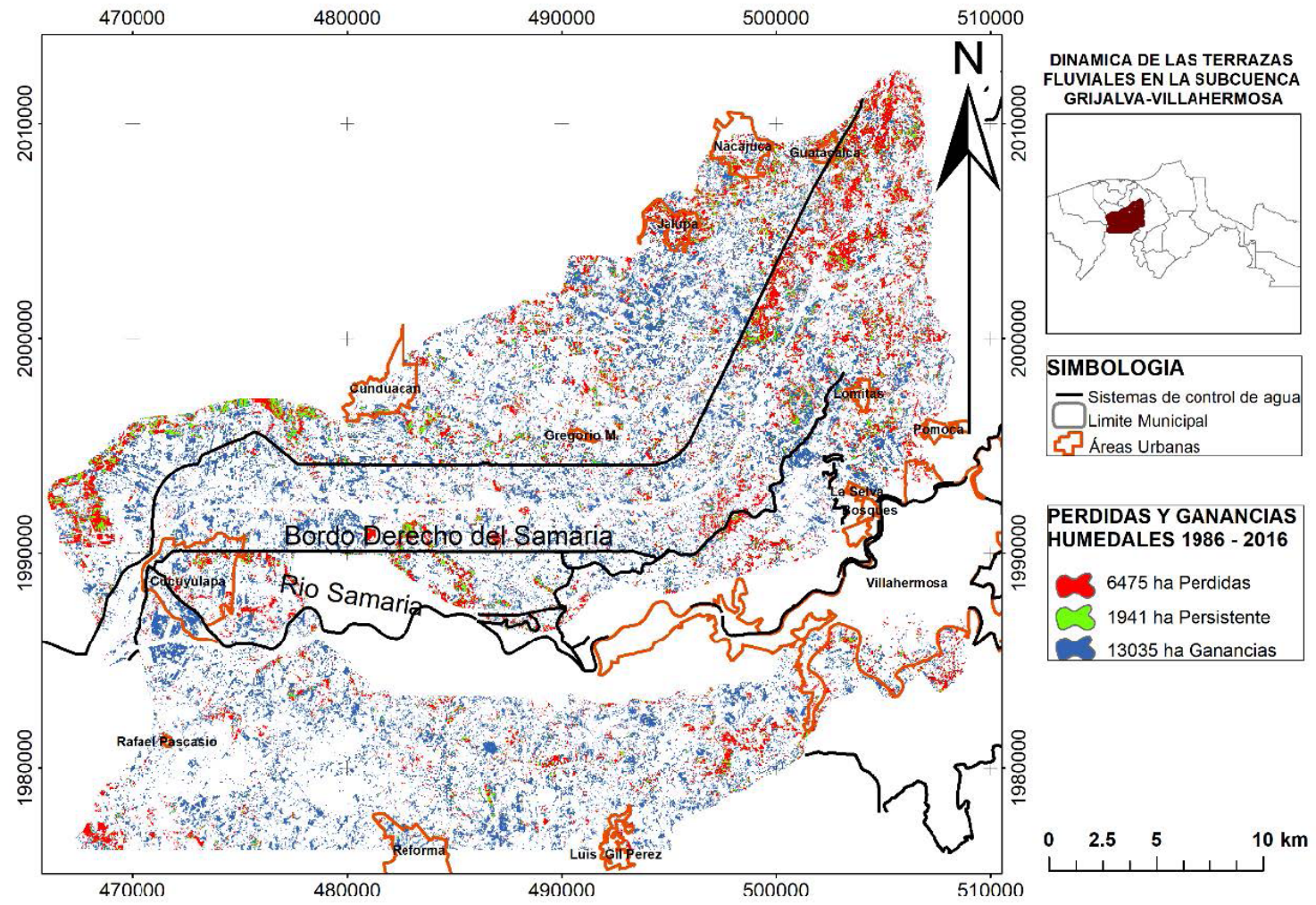

Figura 5 Mapa de Pérdidas y Ganancias de Humedales del Periodo 1986-2016. 
de área, los humedales han prosperado, aumentando su área de distribución principalmente en la zona sur de la Subcuenca debido a un mayor porcentaje de humedad en el suelo y áreas que están siendo sujetas a procesos de anegamiento en las zonas bajas debido a los sistemas de control de agua. En particular, puede observarse que la vegetación (Figura 6) registró cambios significativos con una pérdida de cobertura de 25321 ha. Sin embargo, las áreas donde persistió y se registraron ganancias es mayor (38456 ha).

En la Figura 7 se pueden observar la relación de pérdidas y ganancias estimadas en el periodo de análisis de la infraestructura antrópica. Como respuesta a los sistemas de control de agua, se registró un crecimiento significativo (3242 ha) de zonas urbanas en la Subcuenca GrijalvaVillahermosa. Sin embargo, las pérdidas rebasan ligeramente a las ganancias (3793 ha), debido a que el crecimiento de zonas urbanas se ha dado en áreas donde no se inundaba constantemente, en particular en la zona sur de la Subcuenca, lo cual puede indicar un aumento en la vulnerabilidad de la población a las inundaciones.

\section{Discusión y Conclusiones}

Las actuaciones en la Subcuenca GrijalvaVillahermosa, tanto en la llanura de inundación como en el propio cauce, durante décadas han sido diversas: construcciones, extracciones, rellenos, bordos, compuertas, entre otras (Tudela, 1992; Conesa y Pérez, 2014). Sin embargo, el desarrollo económico en la Subcuenca se ha visto deteriorado por la constante de inundaciones, las cuales son producto de la vocación natural del suelo de la llanura de Tabasco, así como a eventos prolongados de precipitación resultado de procesos meteorológicos particulares de la zona sureste del Golfo de México y a la contribución de los sistemas de control de agua en la alteración de régimen natural de los escurrimientos por los cambios de uso de suelo que modificaron sustancialmente las condiciones ambientales de la Subcuenca Grijalva-Villahermosa (Huerta y Salado, 2012; Plascencia-Vargas et al., 2014).
El análisis de tendencia de cambio para el periodo analizado 1986-2016 registró un impacto significativo con base en los criterios ambientales establecidos para este estudio: vegetación, humedales, suelo, cuerpos de agua e infraestructura antrópica. El resultado observado de las modificaciones en la dinámica natural de la Subcuenca ejercida principalmente por el cambio de uso de suelo representando por la cobertura de vegetación, así como las derivadas de la instalación de sistemas de control de agua y, por consecuencia, el desarrollo de zonas urbanas bajo la percepción de seguridad que ofrecen estos sistemas de control, ha incrementado la vulnerabilidad de zonas que ocasionalmente se inundaban a condiciones de riesgo de inundación frecuente o permanente. Otros estudios, como el de Arreguín-Cortés et al. (2014) y Ramos-Reyes et al. (2016) discuten resultados similares cuando el análisis de estos factores es planteado desde la perspectiva del cambio climático, indicando que es necesario diseñar un ordenamiento territorial congruente con la vocación del suelo y la dinámica de la llanura.

Por otra parte, los sistemas de control de agua han ejercido un impacto significativo en los humedales de la Subcuenca. Por sus características bióticas y la regulación hidrológica que ejercen en las llanuras inundables, los humedales son los ecosistemas más susceptibles al deterioro por factores antrópicos y la alteración de la dinámica hidrológica, factores que inciden en la pérdida de biodiversidad y reducción de áreas de distribución, por lo tanto, en la capacidad de regulación hídrica de esos ecosistemas. En este trabajo se muestra que a pesar de haberse registrado una pérdida importante (6475 ha), el incremento en suelos anegados en las zonas bajas de la Subcuenca Grijalva-Villahermosa ha favorecido una dispersión y permanencia de los humedales, de tal manera que su valor y función ecológica natural en la captación de agua se mantiene (Barba-Macías et al., 2006; Flórez et al., 2016). Sin embargo, se requieren de estudios de fragmentación paisajística de estos ecosistemas que permitan el análisis de su transformación y la prestación de servicios ambientales (Arias-García y Gómez-Zotano, 2015; Mateus Baez y Caicedo Castañeda, 2016). 


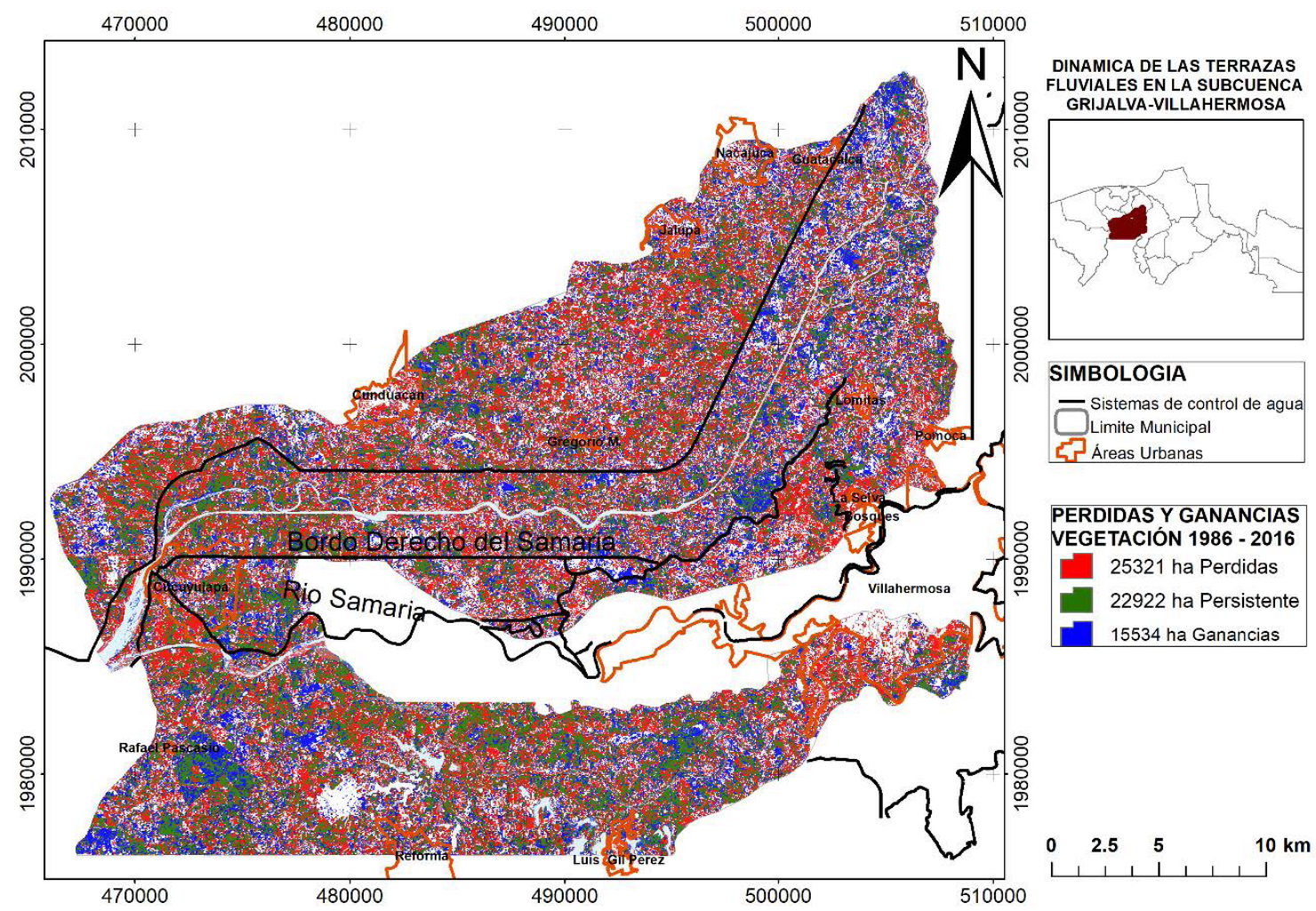

Figura 6 Mapa de Pérdidas y Ganancias de Vegetación del Periodo 1986-2016.

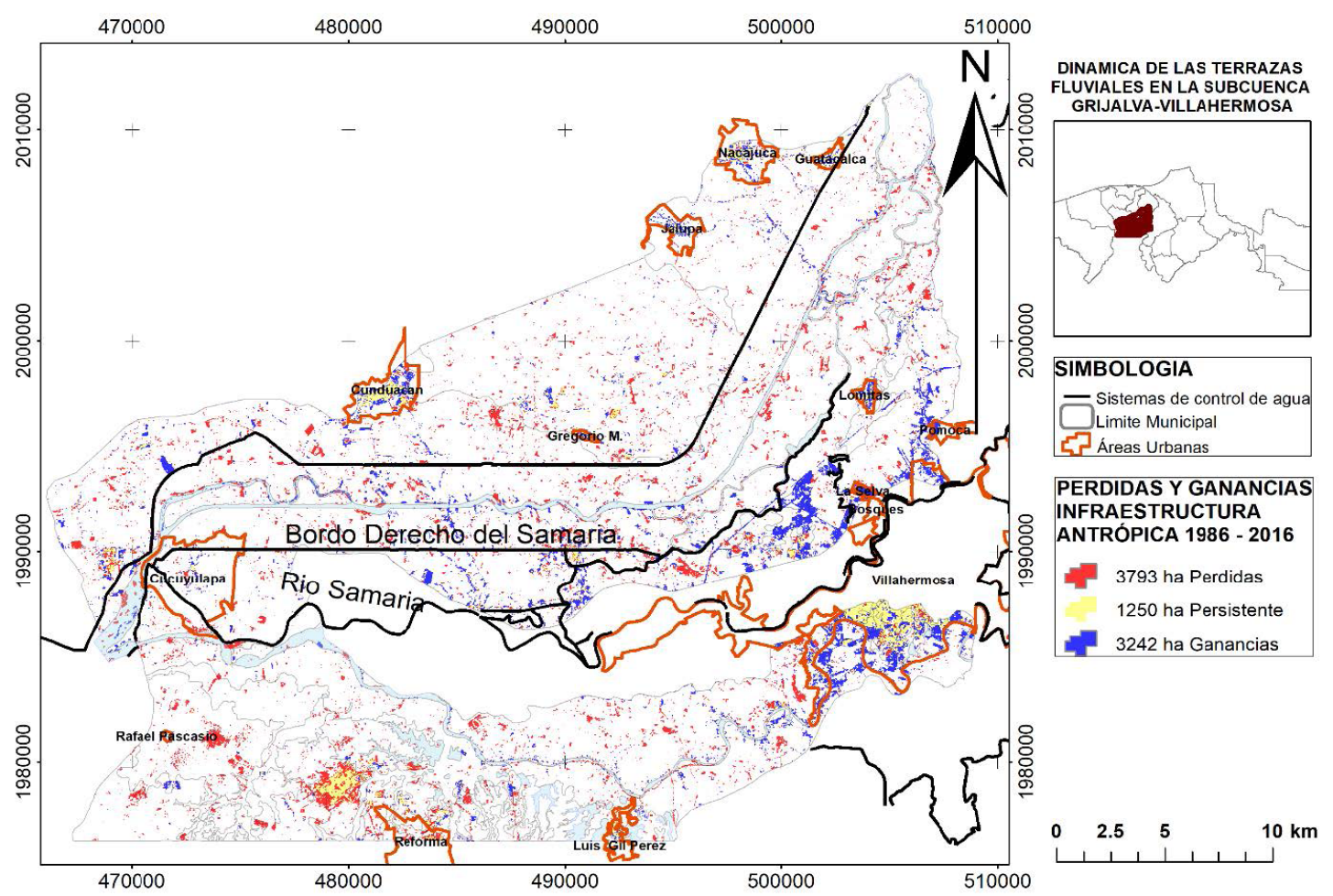

Figura 7 Mapa de Pérdidas y Ganancias de Infraestructura Antrópica de los años 1986-2016. 
El desarrollo urbano, por su parte, ha propiciado la ocupación de terrenos inundables y de zonas de regulación hídrica, lo cual impide el escurrimiento natural del agua cuando los ríos desbordan. Consecutivamente, esto ha influenciado el ascenso de los niveles de azolvamiento de los ríos por acumulación de sedimentos que reducen la capacidad de transportación libre del agua, contribuyendo a los procesos de inundación (CONAGUA, 2005; Palomeque de la Cruz et al, 2017). La dinámica actual en la Subcuenca Grijalva-Villahermosa muestra un comportamiento de impacto generado por cambios en el uso del suelo debido a la percepción de seguridad provista por los sistemas de control de agua y al desarrollo de zonas urbanas, incrementándose de esta manera procesos de cambio de uso de suelo relacionado a actividades antrópicas de carácter productivo. Estos cambios han ocasionado que zonas que no registraban inundaciones periódicas o prolongadas sean afectadas con mayor frecuencia, resultando en el abandono de las viviendas y favoreciendo la recuperación de espacios por los humedales (Requena, 2015).

Capdepont-Ballina y Marín-Olán (2014) discuten que Tabasco es un territorio de características rurales que ha sido transformado hacia lo urbano por el desarrollo de la economía basada en el petróleo, ocasionando un incremento en el índice demográfico.

En la necesidad de salvaguardar, por lo tanto, a la población y a la industria de los procesos de inundación, lo sistemas de control de agua han contribuido a una percepción de seguridad que ha tenido como resultado una expansión de las zonas urbanas hacia terrenos no aptos para la construcción de viviendas, ya que son generalmente zonas de amortiguamiento o terrenos inundables (Cuanalo et al., 2016). Las intervenciones antrópicas, de acuerdo con los resultados del presente trabajo, son el resultado de una planificación espacial inapropiada. Por una parte, los sistemas de control de agua no han mejorado el flujo de las escorrentías o las avenidas importantes como estrategia de mitigación de inundaciones, dando lugar a una mayor retención de agua en los sistemas ambientales y, en consecuencia, esto ha originado cambios de uso de suelo sobre las terrazas fluviales de la Subcuenca Grijalva-Villahermosa por la falta de aplicación de un plan de ordenamiento urbano.

$\mathrm{El}$ análisis de tendencia de cambio resultante de este diagnóstico muestra el impacto negativo de los sistemas de control de agua y el potencial de identificación de espacios inundables a través de métodos de análisis multi-temporal como Markov en el que los sistemas ambientales presentaron una tendencia de cambio a infraestructura antrópica en el periodo analizado entre 1986 y 2016. Por lo tanto, el presente trabajo es una contribución al análisis del proceso de inundación en planicies bajas mediante sistemas de información geográfica y puede ser integrado en simulaciones de eventos de inundación.

\section{Agradecimientos}

Agradecemos a la Delegación Local del Instituto Nacional de Estadística, Geografia e Informática (INEGI) por la información proporcionada diligentemente.

\section{Referencias}

Arreguín-Cortés, F. I., Rubio-Gutiérrez, H., Domínguez-Mora, R., Luna-Cruz, F. D., 2014, Análisis de las inundaciones en la planicie tabasqueña en el periodo 19952010: Tecnología y Ciencias del Agua, 5 (3), 05-32.

Arias-García, J., Gómez-Zotano, J., 2015, La planificación y gestión de los humedales de Andalucía en el marco del Convenio Ramsar: Investigaciones Geográficas, 63 117-125. https://doi.org/10.14198/ingeo2015.63.08

Barba-Macías, E., Rangel-Mendoza, J., RamosReyes, R., 2006, Clasificación de los humedales de Tabasco mediante sistemas de información geográfica. Ecosistemas y Recursos Agropecuarios, Universidad Juárez Autónoma de Tabasco. Villahermosa, Tabasco 102-107. 
Brown,A.G., 1996,Floodplainpalaeoenvironments, en Anderson, M.G., Walling, P.D. and Bates, P.D. (eds.): Floodplain Processes John Wiley \& Sons, Chichester, 95-138

Capdepont-Ballina, J. L., Marín-Olán, P., 2014, La economía de Tabasco y su impacto en el crecimiento urbano de la ciudad de Villahermosa (1960-2010): LiminaR, 12(1), 144-160. http://dx.doi.org/10.29043/ liminar.v12il.330

Comisión Nacional del Agua (Conagua), 2005, Plan Hidráulico Estatal. México: Conagua. 195.

Conesa García, C., Pérez Cutillas, P., 2014, Alteraciones geomorfológicas recientes en los sistemas fluviales mediterráneos de la Península Ibérica. Síntomas y problemas de incisión en los cauces: Revista de Geografía Norte Grande, 59, 25-44. http://dx.doi. org/10.4067/S07 18-34022014000300003

Cuanalo, O. A., Amaya, R. J. G., Lemus, L. J. Q., 2016, Problemas Geotécnicos en Edificaciones Desplantadas sobre Depósitos Aluviales en Llanuras y Penillanuras de Inundación: Revista Ingenio. Universidad Francisco de Paula Santander Ocaña (UFPSO), Vía Acolsure, 12(1), 23-31.

Flórez, C., Estupiñán-Suárez, L. M., Rojas, S., Aponte, C., Quiñones, M., Acevedo, Ó., Jaramillo, Ú., 2016, Identificación espacial de los sistemas de humedales continentales de Colombia: Biota Colombiana 17(1) 45-55. https://doi.org/10.21068/bc.v17isupl. $\% 20$ 1.409

Guerra-Martínez, V., Ochoa-Gaona, S., 2008, Evaluación del programa de manejo de la Reserva de la Biosfera Pantanos de Centla en Tabasco, México: Universidad y Ciencia, 24 (2), 135-146.

Huerta, H. V., Salado, A. I. A., 2012, Modelación Espacio-Temporal de las Precipitaciones Máximas y Estimación de Riegos de Inundación en el Estado de Tabasco, en Martínez Ruiz, R., Ramírez-Valverde, B., Rojo Martínez G. E. (Eds.): Recursos Naturales y Contaminación Ambiental, Cuerpo Académico Desarrollo Sustentable. Universidad Autónoma Indígena de México. $1-26$.
Howard, A.D., 1996, Modelling Channel evolution and Floodplain morphology, en Anderson, M.G., Walling, P.D. and Bates, P.D. (eds.): Floodplain Processes John Wiley \& Sons, Chichester, 15-62.

Instituto Nacional de Estadística, Geografía e Informática., (INEGI), 2001, Síntesis de Información geográfica del estado de Tabasco. (en línea), México. Disponible en: http: internet.contenidos.ingei.com. $\mathrm{mx} / \mathrm{contenido/productos/prod \_ serv/}$ contenidos/espanol/bvinegi/productos/ historicos/2104/702825223939/ 702825223939_6.pdf,

consultado $31 / 10 / 2015$

Mateus Baez, F. A., Caicedo Castañeda, Y. L., 2016, Efecto de la transformación del paisaje sobre la prestación del servicio ecosistémico de provisión de hábitat del humedal "El Tunjo" (Bogotá-Colombia) de 1940 a 2014: Bogotá, Colombia, Universidad de Ciencias Aplicadas y Ambientales (UDCA), Tesis de licenciatura, $91 \mathrm{p}$.

Nanson, G.C., Croke, J.C, 1992, A genetic classification of floodplains: Geomorphology 4(6), 459-486. https://doi. org/10.1016/0169-555x(92)90039-q

Ortíz-Pérez M. A, Siebe, C., Cram, S., 2005, Diferenciación ecogeográfica de Tabasco. Biodiversidad del estado de Tabasco, México: Instituto de Biología, UNAM-CONABIO. 305-322.

Ortiz-Solorio, L., M. Anaya G. y J.W. Estrada B., 1995, Evaluación, cartografía y políticas preventivas de la degradación de la tierra. México: Colegio de Posgraduados, Universidad Autónoma de Chapingo y CONAGyT. $160 \mathrm{p}$.

Palomeque de la Cruz, M. Á., Galindo Alcántar, A., Pérez Sánchez, E., de Jesús Sánchez, A., Escalona Maurice, M. J., 2017, Modelos geomáticos con base en transición para el análisis espacial en Villahermosa, Tabasco: Revista Mexicana de Ciencias Agrícolas, 8(2), 253-267. https://doi.org/10.29312/ remexca.v8i2.48 
Paegelow, M., Camacho Olmedo, M. T., Menor Toribio, J., 2003, Cadenas de Markov, evaluación multicriterio y evaluación multiobjetivo para la modelización prospectiva del paisaje: Revista Internacional de Ciencia y Tecnología de la Información Geográfica. GeoFocus 3, 22-44

Plascencia-Vargas, H., González-Espinosa, M., Ramírez-Marcial, N., Álvarez-Solís, D., Musálem-Castillejos, K., 2014., Características físico-bióticas de la cuenca del río Grijalva, en González-Espinosa M, Brunel Manse MC (eds). Montañas, pueblos y agua: dimensiones y realidades de la cuenca Grijalva: México, Editorial Juan Pablos, 29-79.

Ramos-Reyes, R., Palma-López, D. J., OrtizSolorio, G. A., Ortiz-García, C. F., DíazPadilla, G., 2004, Cambios de uso de suelo mediante técnicas de sistemas de información geográfica en una región cacaotera: Terra Latinoamericana. Sociedad Mexicana de la Ciencia del Suelo, 22 (3), 267-278.

Ramos Reyes, R., Zavala-Cruz, J., Gama Campillo, L. M., Pech Pool, D., Ortiz Pérez, M. A., 2016, Indicadores geomorfológicos para evaluar la vulnerabilidad por inundación ante el ascenso del nivel del mar debido al cambio climático en la costa de Tabasco y Campeche, México. Boletín de la Sociedad Geológica Mexicana, 68 (3), 581-598. http:/ / dx.doi.org/10.18268/BSGM2016v68n3a12

Requena, López L., 2015, Evaluación EspacioTemporal de Impactos Acumulativos en la Subcuenca Villahermosa: Tabasco, México, Universidad Juárez Autónoma de Tabasco. Tesis Maestría, 26-56.

Rojas, O., Mardones, M., Arumí, J. L., Aguayo, M., 2014, Una revisión de inundaciones fluviales en Chile, período 1574-2012: causas, recurrencia y efectos geográficos: Revista de Geografía Norte Grande. 57, 177-192. http://dx.doi.org/10.4067/ S07 18-34022014000100012
Schumm, S.A., 1993, River response to Base Level Change: Implications for Sequence Stratigraphy: The Journal of Geology, 101, 279-294. https://doi.org/10.1086/648221

Toledo Sánchez, H., 2011, Fragilidad, vulnerabilidad y riesgo en la Cuenca baja del Sistema Grijalva-Usumacinta: México, Instituto Politécnico Nacional. México, Tesis Doctoral, 143 p.

Tudela, F., 1992, La modernización forzada del trópico: El caso de Tabasco, proyecto integrado del Golfo, en Tudela, F. (ed), Megabase Agropecuaria, Alianza SIDALC: Tabasco, México, 475 p.

Vidal, C., Romero, H., 2010, Efectos ambientales de la urbanización de las cuencas de los ríos Bíobío y Andalién sobre los riesgos de inundación y anegamiento de la ciudad de Concepción, en: Pérez, L. e Hidalgo, R. (eds) Concepción metropolitano (AMC). Planes, procesos y proyectos, serie GEOlibros: Santiago de Chile, Pontificia Universidad Católica de Chile. Cap. 1 1-19

Zavala Cruz, J., Salgado García, S., Marín Aguilar, Á., Palma López, D. J., Castelán Estrada, M., Ramos Reyes, R. (2014). Transecto de suelos en terrazas con plantaciones de cítricos en Tabasco: Ecosistemas y recursos agropecuarios, 1(2), 123-137.

Zavala-Cruz J., Jiménez-Ramírez, R., PalmaLópez DJ. Bautista-Zuñiga, F. Gavi-Reyes F., 2016, Paisajes geomorfológicos: Base para el levantamiento de suelos en Tabasco, México: Ecosistemas y Recursos Agropecuarios. 3 (8), 161-171.

Zinck, J. A. 2016, The Geomorphic Landscape: Classification of Geoforms: Geopedology 101-125. https://doi. org/10.1007/978-3-319-19159-1_7 